\title{
Webquest como Escenario para El Aprendizaje Colaborativo y El Desarrollo de Competencias Digitales
}

\section{WebQuest as a Setting for Collaborative Learning and The Development of Digital Skills}

\author{
Elena Katayama Cruz ${ }^{1}$ \\ https://orcid.org/0000-0002-8874-2994 \\ Universidad César Vallejo, Perú \\ John Alexander Rojas Montero ${ }^{2}$ \\ https://orcid.org/0000-0003-1109-1262 \\ Universidad Pedagógica Nacional, Colombia
}

Recibido: 25-07-2021

Aceptado: 23-12-2021

\section{Cita Recomendada}

Katayama, E. y Rojas, J. (2021). WebQuest como escenario para el aprendizaje colaborativo y el desarrollo de competencias digitales. Hamut'ay, 8(3), pág. 58-65.

http://dx.doi.org/10.21503/hamu.v8i3.2332

\begin{abstract}
Resumen
Los tiempos por los que transcurre la humanidad han provocado la implementación acelerada de la educación remota a través de las Tecnologías de la Información y la Comunicación, generándose la necesidad de desarrollar habilidades digitales por parte de los docentes y los estudiantes. En este tipo de procesos se tiene la WebQuest como e-actividad que posibilita el desarrollo de estrategias para el logro de aprendizajes; es por ello que se realiza una exploración en la literatura acerca de este tipo de actividades en el campo educativo desde los procedimientos implementados para su creación, las experiencias de aprendizaje que propicia en los estudiantes, la identificación de su estructura y las ventajas de su aplicación, adecuación o innovación en el desarrollo y promoción del aprendizaje colaborativo. En conclusión, la WebQuest propone varias acciones secuenciales y sistemáticas orientadas al desarrollo del pensamiento crítico, creativo y el trabajo en equipo, para aprender de manera reflexiva.
\end{abstract}

Palabras Clave: WebQuest, e-actividad, habilidades digitales, aprendizaje colaborativo, TIC.

\footnotetext{
1. Estudiante del Doctorado en Educación de la Universidad César Vallejo-SP, Magíster en Educación, Licenciada en Educación. Estudiante adscrita al proyecto PIPD, Innovación docente y uso de las Tecnologías de la Información y Comunicación en el proceso de enseñanza-aprendizaje y al Grupo de Investigación en Psicología, educación y tecnologías-IPET-UCV.

2. Doctor en Educación, Magíster en Tecnologías de la Información Aplicadas a la Educación, Magíster en Gestión de Organizaciones, Especialista en Entornos Virtuales de Aprendizaje, Ingeniero de Sistemas; co-investigador del Grupo de Investigación en Psicología, Educación y Tecnologías-IPET de la Universidad César Vallejo. jarojas@pedagogica.edu.co
} 


\begin{abstract}
The times through which humanity passes have caused the accelerated implementation of remote education through Information and Communication Technologies, generating the need to develop digital skills on the part of teachers and students. This type of process, we have the WebQuest as an e-activity that enables the development of strategies to achieve learning; That is why an exploration is carried out in the literature about this type of activities in the educational field from the procedures implemented for its creation, the learning experiences that it fosters in students, the identification of its structure and the advantages of its application, adaptation or innovation in the development and promotion of collaborative learning. In conclusion, the WebQuest proposes several sequential and systematic actions aimed at the development of critical and creative thinking and teamwork, to learn in a reflective way.
\end{abstract}

Key words: WebQuest, e-activity, digital skills, collaborative learning, ICT.

\section{Introducción}

Los vertiginosos cambios y progresos de las Tecnologías de la Información y la Comunicación (TIC), siguen produciendo transformaciones en el contexto educativo, particularmente en las didácticas empleadas por los profesores, quienes deben planificar diversas formas de intervención durante los procesos de formación que orientan, para los cuales han de considerar diversas estrategias que combinan actividades, recursos y contenidos que son permeados por tecnologías como plataformas, correos, blogs o WebQuest, como soporte de la producción de conocimiento a través de la computación social (García, 2017). En estos días, las TIC aparecen en entidades $u$ organizaciones con más frecuencia y su uso va en aumento, situación a la que no es ajena la escuela, y ante la que se requiere de profesores con habilidades para seleccionar y manejar estas tecnologías con el fin de hacerlas parte de las estrategias pedagógicas que emplean en las experiencias curriculares que plantean (Ccoa y Alvites-Huamaní, 2021; Holguín, F., Holguín, E. y García, 2020).

En Perú, las instituciones educativas de educación básica y superior han implementado la educación remota a partir del uso de la Web, de recursos tecnológicos y entornos digitales (Hidalgo, 2020; Minedu, 2020; Alvites-Huamaní, 2020; Pineda et al., 2021; Soto, Valdivieso y Talledo, 2021). Estas tecnologías se volvieron esenciales para favorecer el proceso de enseñanza y mantener la comunicación de forma permanente y creativa entre docentes y estudiantes, de allí la necesidad de conocerlas y dominarlas (Montenegro, 2020). En este contexto emerge la WebQuest como posibilidad de innovación en el ámbito educativo que propicia el aprendizaje al brindar motivación y refuerzo positivo en los estudiantes, por lo que desde el quehacer docente ha de buscarse garantizar la conexión entre las TIC y las necesidades de aprendizaje de nuevas generaciones de estudiantes, de manera que se organice su integración al aula de clase (Chalco y Quispe, 2018; Gómez, 2019; MartínezBorreguero et al., 2020; Calizaya, 2021; Turpo, 2021).

A pesar de lo anterior, es habitual encontrar prácticas de adolescentes y jóvenes estudiantes poco adecuadas, como es el caso de la búsqueda de información en Internet, ya que con solo ingresar algunas palabras, seleccionan lo primero que se les presenta sin razonar en la confiabilidad de la misma, lo que puede atribuirse al escaso desarrollo de habilidades digitales que los lleva a no estar lo suficientemente preparados para el mundo digital (Ulu y Ulusoy, 2019; Silva, 2020); por esta razón es necesario potenciar el uso de las TIC con intervenciones innovadoras que mejoren la enseñanza de los profesores, que atiendan de manera diferenciada las necesidades de los estudiantes y que promueva el trabajo colaborativo y el pensamiento crítico, y no la reproducción automática de conceptos apoyados con la tecnología (Cóndor, 2019; Peris, 2020; 
Torres et al., 2020; Quispe, 2021).

En los escenarios hasta aquí descritos, se identifican insuficiencias en los docentes, primero en la adquisición de habilidades para el uso adecuado de Internet y la Web, y segundo en adecuar los recursos digitales a estrategias que respondan a las intenciones del currículo a través de actividades y contenidos que incentiven la exploración y la investigación de otras didácticas para que el estudiante adquiera diversas competencias (Tran, 2018). Es de recordar que la aplicación de recursos digitales en actividades de enseñanza y aprendizaje, requiere planificar su aplicación con el propósito de coadyuvar a la construcción de nuevos saberes; en ese sentido, la WebQuest es una e-actividad que propicia el diseño de experiencias de aprendizaje que van en concordancia con un currículo enfocado hacia la organización activa de la indagación, el mejoramiento de los aprendizajes, y el fortalecimiento de la interacción entre profesores y estudiantes (De Souza et al., 2017).

En este trabajo, se analiza la WebQuest respecto a la promoción de aprendizajes centrados en las necesidades y motivaciones de los estudiantes, por lo que se recurre a la revisión de documentos que consolidan su uso en experiencias formativas.

\section{Método}

Como método de investigación se aplica un enfoque cualitativo documental, basado en la revisión teórica de fuentes de información científicas registrados en la base de datos Scopus, Dialnet, Redalyc, Scielo, Latindex y repositorios universitarios; las fuentes se seleccionaron considerando artículos de revistas, libros en formato digital, trabajos de grado y comunicaciones de congresos y conferencias científicas, a través de palabras claves como la variable WebQuest, antecedentes y experiencias relacionadas a su aplicabilidad en la enseñanza y aprendizaje, desarrollo de capacidades, mejora de aprendizajes en la educación, desarrollo de entornos virtuales, y habilidades en el uso de tecnología e información, y otras temáticas relacionados al objetivo. La información se organiza y analiza con rigurosidad para ser interpretada, siendo fundamental contar con fuentes de autores primigenios y explicación de manera objetiva y argumentada que cuestiona en profundidad la revisión bibliográfica, discusión y conclusión de los estudios citados (Gómez, 2010).

\section{Una exploración a la WebQuest}

Para su autor, Bernie Dodge, la WebQuest es una actividad de investigación, creada de forma intencional, que presenta información procedente de Internet y propicia la interrelación de los estudiantes con el objetivo de desarrollar procesos cognitivos relacionados con el análisis, la síntesis y la evaluación (Adell, 2015). Después de más de dos décadas, es vista como una e-actividad de enseñanza constructivista centrada en el estudiante, que hace uso de teorías, métodos, estrategias, plataformas, blogs, comunidades sociales y otras fuentes obtenidas de Internet, previamente seleccionados $y$ contextualizados por los docentes de acuerdo a una estructura (Tabla 1).

Tabla 1 Estructura de una WebQuest
Básico, descrita por Dodge (Adell, Mengual y Roig, 2015)
Aportes a la estructura original
1. Introducción: prepara el escenario y aporta cierta información
Trabajo cooperativo, todos los participantes del equipo asumen responsabilidades para realizar la tarea y socializar (Adell, 2004).
2. Tarea: realizable y retadora

3. Recursos tecnológicos:

4. Descripción: presentación

5. Conclusión: argumento

Fuente:Adaptado de (Adell, 2004; Adell, Mengual y Roig, 2015; González soportes de información relacionados con la tarea tomados total o parcialmente de Internet del proceso que los estudiantes deben seguir para ejecutar la tarea final de la tarea aplicable a

otra experiencia y De León, 2011).

Existen experiencias exitosas con WebQuest con ciertas variantes en su estructura, aparte de considerar: introducción, tarea, proceso, recursos, evaluación, conclusión, agregan otros apartados como: recursos didácticos para el profesor, orientación dirigida a estudiantes e información de interés (González y De León, 2011). 
Esta e-actividad, además permite motivar y guiar el trabajo investigador del estudiante, y la consecución de los aprendizajes con la participación activa del estudiante (Ulu y Ulusoy, 2019); asimismo, propicia el uso de recursos Web fáciles de utilizar por lo que no necesariamente requieren del acompañamiento del docente. En la Figura 1 se muestran diversos tipos de WebQuest que se pueden seleccionar de acuerdo al interés y temática que tenga planificada el docente (Martínez y Déniz, 2018).

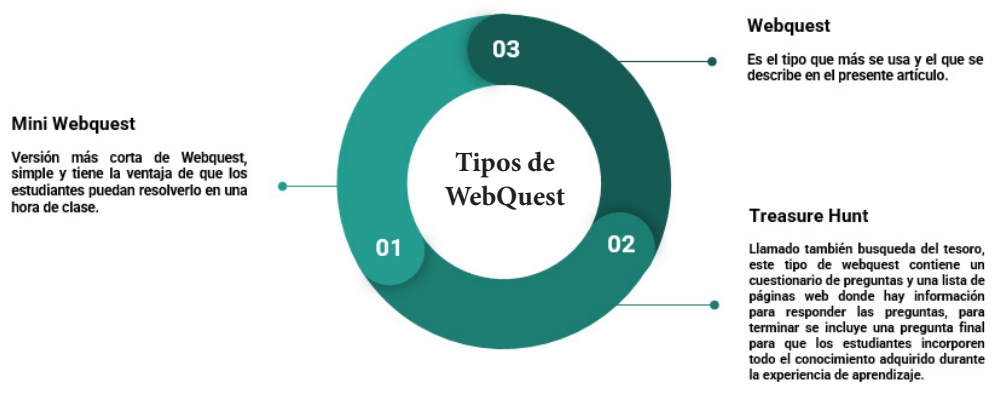

Figura 1. Tipos de WebQuest.

Fuente: Adaptado de Martínez y Déniz (2018).

En el contexto actual, la presencia de una e-actividad como la WebQuest resulta interesante para el desarrollo de pensamientos cognitivos de orden superior y la autonomía en el aprendizaje de los estudiantes (Salic-Hairulla et al, 2018). Al estar diseñada para su aplicación en la Web, se adapta a las innovaciones tecnológicas difundidas masivamente por medios sociales como parte de la construcción de recursos integrados con un enfoque por tareas (Lim y Hernández, 2007). En la Figura 2 se presentan las características específicas de la estructura ordenada y sistemática de la WebQuest que resalta su importancia como estrategia en el proceso de aprendizaje (Burbano, 2020).

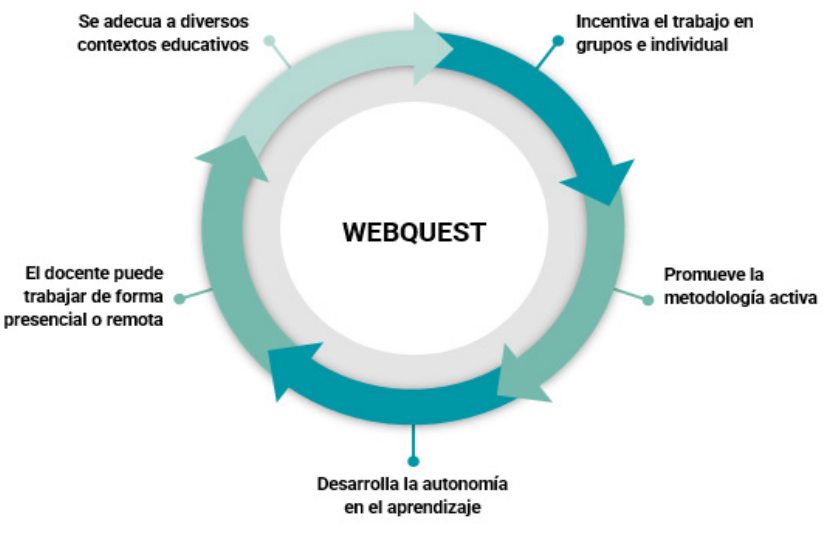

Figura 2: Características de la Webquest Fuente: Burbano (2020). Adaptación propia.

\section{WebQuest en educación}

La WebQuest como cualquier otra e-actividad educativa, requiere de un plan de intervención pedagógico y la responsabilidad del docente para adecuarlas didácticamente antes de aplicarlas en una experiencia de aprendizaje significativa; en ese sentido, su preparación para los estudiantes debe prever planes con acciones que provoquen la indagación en Internet; el análisis y la comprensión de escritos, imágenes, audios y videos; el acompañamiento del docente para evitar la reproducción de contenidos y motivar al estudiante a formular una tarea propia; la valoración del proceso educativo por parte de los estudiantes al finalizar su desarrollo (De Souza et al., 2017). Asimismo, experiencias que involucran la WebQuest evidencian mejoras en los aprendizajes de los estudiantes, efectos agradables y satisfactorios para los profesores y estudiantes; además de favorecer los procesos cognitivos a través de círculos de estudio, debates, indagación, proyectos, exposición de ideas a través de Internet (Sahin y Huri, 2016).

La WebQuest puede potenciarse a través de OKMindmap, Scratch o Facebook para propiciar el desarrollo de habilidades colaborativas en los estudiantes y las competencias curriculares mediante acciones dinámicas, críticas y reflexivas; siempre que respondan a un plan con experiencias de aprendizajes adecuadamente diseñadas (Diem et al, 2018). Además, en un estudio realizado por Sosa (2016) se concluye que se favorecen los procesos formativos a partir de la motivación y el trabajo colaborativo (Figura 2).

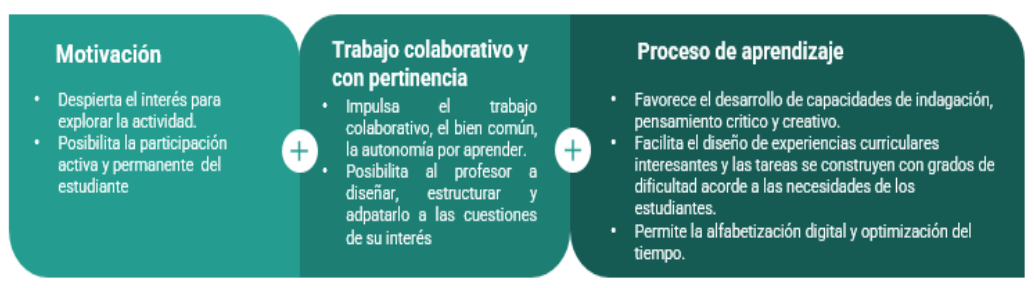

Figura 2: Ventajas de webquest en el aprendizaje

Fuente: Adaptado de Sosa (2016)

En consecuencia, las e-actividades en cualquiera de sus formas aunado a la planificación 
pedagógica con estrategias que gestionen la autonomía de los estudiantes, serán eficaces siempre que los profesores no propongan tareas tradicionales (Chong-Baque, 2020). Como se ha indicado, el docente tiene a disposición los recursos de la Web, los cuales selecciona y emplea para favorecer el aprendizaje del estudiante a través de la indagación, el descubrimiento, el fortalecimiento de propuestas curriculares mediadas por las TIC, bajo la premisa que el estudiante sea el centro activo y autónomo de su saber (Serna y Santillán, 2021). Por otro lado, es responsabilidad del docente, implementar actividades auténticas cementadas en la teoría del conocimiento constructivista, en donde los estudiantes tengan oportunidades para desplegar habilidades a través de las TIC, para organizarse y trabajar en equipo, para apoyarse mutuamente, para aprender de manera activa (Corujo-Vélez, Gómez-del-Castillo y Merla-Gonzáles, 2019). En ese sentido, el rol del docente es vital para romper las barreras tecnológicas ante los cambios y avances, es decir, se requiere que reevalúen la forma como tradicionalmente han realizado los procesos de enseñanza, e incorporen recursos digitales que inviten a la discusión para evitar el fomento de bajos niveles de aprendizaje (Da Costa et al., 2018).

De igual importancia, es la organización de la WebQuest, para favorecer experiencias de aprendizaje individuales y grupales que refuercen el trabajo cooperativo y colaborativo con el fin de desarrollar el pensamiento complejo y aprovechar el acceso a la información en Internet que tienen docentes y estudiantes, quienes aúnan esfuerzos para comprender y originar un producto, proceso o tarea particular apoyados en las TIC generadoras de sensaciones de satisfacción y disfrute en el aula de clase (Rocu, Blández y Sierra, 2018; Regueira, González-Villa y Gewerc, 2021).

Además del acceso a la información, los estudiantes deben distinguirla, ordenarla y transformarla para alcanzar un aprendizaje propio y auténtico, lo que convierte a la WebQuest en un escenario que potencia el fortalecimiento de diversas habilidades para el aprendizaje como: (a) las del pensamiento crítico, la creatividad, el desarrollo de la lectura y la escritura; (b) las lingüísticas para comprender un idioma; o (c) las informáticas para la apropiación de las TIC. Para ello, las tareas planteadas en una e-actividad pueden darse de forma individual, en parejas o en equipos dependiendo de las indicaciones adoptadas por el docente; las cuales al concluirse permiten conocer a partir de experiencias de aprendizaje objetivas, interactuar a partir del trabajo en equipo con actitudes positivas y de expresar a partir del intercambio de ideas durante la discusión en clase, lo que en consecuencia, motiva a los estudiantes (Adanan, H., Adanan, M. y Herawan, 2020).

Los estudios analizados sobre la WebQuest, vislumbran resultados positivos en la educación formal de primaria y secundaria, evidenciados en la construcción de conocimiento por parte de los estudiantes, en la participación activa, en el empleo de material basado en proyectos y en situaciones del contexto y, en la integración de profesores de diferentes áreas curriculares (Czerwinski y Cogo, 2018; Doulgeri y Antoniou, 2018).

\section{Conclusiones}

La WebQuest es una e-actividad que abre el panorama al diseño de escenarios de aprendizaje donde se fortalecen dimensiones epistemológicas, éticas y estéticas, que dan la posibilidad a los estudiantes de conocer, de interactuar y de expresar, como parte de sus procesos formativos en los que obtienen resultados positivos, lo que lleva a visionar un programa curricular para el contexto local fundamentado en su uso frecuente y en el fomento de diversas habilidades y competencias en el contexto digital.

Los escenarios analizados para la WebQuest, muestran que el aprendizaje significativo requiere del trabajo en equipo, de la interacción con pares y profesores, de la realimentación oportuna al momento de cumplir con las tareas formativas propuestas. Esto implica transformaciones en los ámbitos educativos, pedagógicos y didácticos. En los educativos para que las Instituciones de 
Educación Básica y Superior se comprometan con el apoyo al desarrollo de currículos que proponen otras maneras de llevar a los estudiantes a aprender. En lo pedagógico al abordaje de otras metodologías formativas. En lo didáctico al fomento de habilidades digitales como parte de otras técnicas y métodos de enseñar.

Lastareas formativas involucradas en la WebQuest llevan a probar enfoques constructivistas como el aprendizaje basado en proyectos, el aprendizaje basado en la investigación, el aprendizaje basado en problemas, el aprendizaje basado en ejemplos, el aprendizaje multisensorial o el aprendizaje basado en animaciones, entre otros. Estos enfoques requieren de docentes preparados para abordar las Tecnologías de la Información y la Comunicación como territorios digitales en los que estudiantes y profesores toman posturas que los enuncian como sujetos y como colectivo que construyen sus propios conocimientos; en los que el espacio y el tiempo pasan de lo análogo a lo digital, de lo sincrónico a lo asincrónico; en los que se entretejen saberes, relaciones y expresiones; en los que se desarrollan prácticas para el crecimiento intelectual.

Finalmente, los momentos que vivela humanidad, llevan a pensar en las condiciones que se tienen en países como Perú para que propuestas de innovación educativa centradas en las TIC puedan concretarse. Es así, que las Instituciones deben contar con programas de formación docente que potencien sus prácticas pedagógicas y con infraestructuras que mantengan a estudiantes y profesores interconectados. De lo contrario, más que posibilidades, se incrementan brechas sociales digitales que impiden mejorar la calidad educativa en contextos latinoamericanos.

\section{Agradecimiento}

Un especial reconocimiento y agradecimiento por el apoyo al desarrollo de este estudio, como parte del proyecto PIPD, Innovación docentey uso delas Tecnologías de la Información y Comunicación en el proceso de enseñanza aprendizaje con Resolución de Dirección Académica No. 012 2020-DA-UCV. Coordinación del Doctorado en
Educación en la Modalidad Semipresencial de la Escuela de Posgrado de la Universidad César Vallejo y al grupo de investigación en psicología, educación y tecnologías-IPET aprobado con RVI No313-2021-VI-UCV.

\section{Referencias Bibliográficas}

Adanan, H., Adanan, M., \& Herawan, T. (2020). M-WebQuest Development: Reading Comprehension of Senior High School Students in Indonesia. Revista Internacional de tecnologías emergentes en el aprendizaje (iJET), 15(3), 7492. https://doi.org/10.3991/ijet.v15i03.10628

Adell, J. (2004). Internet en el aula: las WebQuest. Edutec. Revista Electrónica de Tecnología Educativa, (17). https://doi.org/10.21556/ edutec.2004.17.530.

Adell, Mengual y Roig, 2015. WebQuest: 20 años utilizando internet como recurso para el aula. Edutec. Revista Electrónica de Tecnología Educativa. (52).

Alvites-Huamaní, C. (2020). COVID-19 Transformaciones a nivel globalizado en educación. Hamut'ay, 7(2), 5-7. http://dx.doi. org/10.21503/hamu.v7i2.2147

Burbano, R. A. (2020). WebQuest como herramienta didáctica en el proceso de enseñanza aprendizaje del área de Ciencias Naturales y Educación Ambiental (Tesis de posgrado). Universidad Internacional de La Rioja, España. https://reunir.unir.net/handle/123456789/9970

Calizaya Limache, C. R. (2021). Estrategias didácticas usando WebQuest y los aprendizajes en Ciencia y Ambiente en el $4^{\circ}$ de Primaria. Institución Educativa Privada William Prescott. Tacna. 2013 (Tesis de maestría). Universidad Nacional "Pedro Luis Gallo", Lambayeque - Perú. Ccoa, F., y Alvites-Huamaní, C. (2021). Herramientas digitales para entornos educativos virtuales. Revista Lex, 19(27), 315-330. http:// dx.doi.org/10.21503/lex.v19i27.2265

Chalco Alcca, A. D., y Quispe Vega, J. H. (2018). Aplicación de la WebQuest, como estrategia de Enseñanza-Aprendizaje, para diseñar Planes de Negocio, en el área de Educación para el Trabajo, con estudiantes del quinto grado de 
Educación Secundaria, Institución Educativa No 40055 Romeo Luna Victoria, distrito Cerro Colorado, provincia Arequipa, 2016 (Tesis de pregrado). Universidad Nacional de San Agustín de Arequipa, Arequipa, Perú.

Chong-Baque, P. G. (2020). Estrategias pedagógicas innovadoras en entornos virtuales de aprendizaje. Dominio de las Ciencias, 6(3), 56-77. http://dx.doi.org/10.23857/dc.v6i3.1274

Cóndor Dorregaray, E. W. (2019). El método de la WebQuest y su relación con la mejora de la calidad del desarrollo del pensamiento crítico en el curso de Ciencia Tecnología y Ambiente en los alumnos del 4to. año de secundaria de la IE Fe y Alegría $N^{\circ}$ 11-Comas (Tesis de maestría). Universidad Nacional de Educación Enrique Guzmán y Valle, Lima, Perú.

Corujo-Vélez, Mª C., Gómez-del-Castillo, Ma T., \& Merla-González, A. E. (2020). Construtivist and collaborative methodology mediated by ICT in Higher education. Pixel-Bit. Revista de Medios y Educación, 57, 7-57. Editorial Universidad de Sevilla. https://doi.org/10.12795/pixelbit.2020. i57.01

Czerwinski G. P. V., \& Cogo, A. L. P. (2018). WebQuest and blog as educational strategies in school health. Revista Gaúcha de Enfermería. 39:e2017-0054. https://doi.org/10.1590/19831447.2018.2017-0054.

Da Costa, F. B., Pereira, C., Dutra, A., Da Rosa, V. F., \& De Oliveira, J. (2018). Uso de recursos podcast e WebQuest no estudo do tema avaliação na educação infantil. Textolivre Linguagem e Tecnologia, 11(2), 92-205. https://doi. org/10.17851/1983-3652.11.2.192-205

De Souza, J. L., Villanueva, J. I., Dutra, A., \& Da Rosa, V. F. (2017). A WebQuest como recurso pedagógico no curso de pedagogía. Textolivre Linguagem e Tecnologia. Belo Horizonte, 10(1), 164-177. https://doi.org/10.17851/19833652.10.1.164-177.

Diem, L., Gi, Y., Ho, W., Thi Thu, H., \& Khang, N. (2018). Developing WebQuest 2.0 model for promoting computational thinking skill. International Journal of Engineering \& Technology, 7 (2.29) 140-144.

Doulgeri, M., \& Antoniou, L. (2018). The effectiveness of a parallel syllabus that uses WebQuests to enhance the New Literacies of 6th grade primary school EFL

learners. Research Papers in Language Teaching and Learning,

9(1), 56-76.

García, O. (2017). Técnicas de computación social e información contextual para el desarrollo de actividades de aprendizaje colaborativo. (Tesis de doctorado), Universidad de Salamanca, España. https://dialnet.unirioja.es/servlet/ tesis? codigo $=155305$

Gómez Segura, R. L. (2019). Los WebQuest y el pensamiento crítico en los alumnos de la institución educativa No 34139 de San Pedro de Pillao-Yanahuanca (Tesis de maestría). Universidad Nacional Daniel Alcides Carrión, Cerro de Pasco, Perú.

Gómez, L. (2010). Un espacio para la investigación documental. Revista Vanguardia Psicológica Clínica Teórica y Práctica, 1(2), 226-233.

González López, I., \& de León y Huertas C. (2011). La WebQuest INVADIV, una propuesta didáctica para fomentar el trabajo cooperativo en el aula. RIED. Revista iberoamericana de educación a distancia, 14(2), 167-188.

Hidalgo Benites, L. E. (2020). Competencias profesionales docentes en la educación remota. CIID Journal, 1(1), 249-270.

Holguín, F. Y., Holguín, E. G. y García, N. A. (2020). Gamificación en la enseñanza de las matemáticas: una revisión sistemática. Telos: revista de Estudios Interdisciplinarios en Ciencias Sociales, 22(1), 62-75. https://doi.org/10.36390/ telos 221.05

Lim, S. L., \& Hernández, P. (2007). The WebQuest: An illustration of instructional technology implementation in MFT training. Contemporary Family Therapy, 29(3), 163-175. https://doi. org/10.1007/s10591-007-9038-y

Martínez, P. E. B., \& y Déniz, S. D. F. (2018). Digital Competence and CLIL: The Use of WebQuests Bilingual Education. e-TEALS: An e-journal of Teacher Education and Applied Language Studies, 9 Special Edition, 153-172.

Martínez-Borreguero, G., Perera-Villalba, J. J., Mateos-Núñez, M., \& Naranjo-Correa, F. L. 
(2020). Development of ICT-Based Didactic Interventions for Learning Sustainability Content: Cognitive and Affective Analysis. Sustainability 2020, 12, 3644. http://dx.doi. org/10.3390/su12093644

Minedu. (2020). Disponen el inicio del año escolar a través de la implementación de la estrategia denominada "Aprendo en casa", a partir del 6 de abril de 2020 y aprueban otras disposiciones. Resolución Ministerial N 160-2020-MINEDU.

Montenegro, D. (2020). Comunicación grupal en Whatsapp para el aprendizaje colaborativo en la coyuntura COVID-19. Hamut'ay, 7 (2), 34-45. http://dx.doi.org/10.21503/hamu.v7i2.2131

Peris Reig, L. (2020). Qué entendemos por innovación: el papel de las TIC. Revista DIM: Didáctica, Innovación y Multimedia, (38).

Pineda Aguilar, W. F., Dávila Talepcio, J., Ortega Chávez, W. y Quispe Sotomayor, L. (2021). Análisis del acompañamiento directivo del trabajo remoto sobre el conocimiento disciplinar y pedagógico del docente de la educación superior peruana mediante mapas cognitivos difusos y método Delphi. Revista Investigación Operacional, 42(3), 334-342.

Quispe Díaz, P. (2021). Estrategia didáctica para desarrollar el aprendizaje colaborativo en los estudiantes del curso de inglés básico en un instituto superior privado de Lima (Tesis de maestría). Universidad San Ignacio de Loyola, Lima, Perú.

Regueira, U., González-Villa, Á., \& Gewerc, A. (2021). Enseñanza en línea bajo pandemia: una experiencia de enseñanza-aprendizaje basada en una WebQuest. En Nuevos retos educativosen la enseñanza superiorfrente al desafío COVID-19 (pp. 784-794). Octaedro Editorial.

Rocu Gómez, P., Blández Ángel, J., \& Sierra Zamorano, M. A. (2018). Construyendo aprendizajes en Expresión Corporal a través de WebQuest: Un estudio de caso múltiple. Revista Complutense de Educación, 30(4), 1013-1029. https://dx.doi.org/10.5209/rced.59905.

Sahin, S., \& Huri, M. (2016). The effect of 5E-learning model supported with WebQuest media on students' achievement and satisfaction. E-Learning and Digital Media, 13(3-4), 158-175. http://dx.doi.org/10.1177/2042753016672903 Salic-Hairulla, M., Agad, L. M., Pitonang,
D. J., Terrado, T. F., \& Yuenyong, Ch. (2018). Implementation of WebQuest in teaching Circulatory System. Journal of Physics: Conference Series, 1340. https://dx.doi. org/10.1088/1742-6596/1340/1/012061

Serna González, P., \& Santillán Galván, R. (2021). WebQuest: marcando el futuro de la educación. Milenaria, Ciencia y Arte, (18), 8-10.

Silva Porras, G. L. (2020). Las WebQuest como recurso tecnológico para el fortalecimiento del trabajo colaborativo (Tesis de pregrado). Pontificia Universidad Javeriana, Bogotá, Colombia.

Sosa Zaragoza, P. (2016). La WebQuest como estrategia de enseñanza-aprendizaje en la formación del auxiliar bibliotecario (Tesis de maestría). Universidad Nacional Autónoma de México, Ciudad de México, México.

Soto Bermúdez, S. S., Valdivieso Chudan, A. R. y Talledo Peña, D. A. (2021). La gestión de la experiencia de la educación remota por emergencia y el nivel de satisfacción en los estudiantes de pre-grado. Caso: Facultad de Derecho de la Pontificia Universidad Católica del Perú (Tesis de pregrado). Pontificia Universidad Católica del Perú, Lima, Perú.

Torres, J. L., Calla, K. M., Castañeda, E. S., Mory, W. y Pumacayo, I. I. (2020). Tecnología de la información y comunicación y las competencias comunicativas en estudiantes universitarios. Revista Espacios, 41, 281-297.

Tran, T. K. T. (2018). Compose the topic of integration to foster the competency of

integrating natural science knowledge for students of Can Tho University's Physics Pedagogy with Webquest_method. Journal of Physics: Conference Series, 1340. https://dx.doi. org/10.1088/1742-6596/1340/1/012035

Turpo Gebera, O. W. (2021). Interacción didáctica y construcción social del conocimiento en Blended Learning: Perú como estudio de caso (Tesis de doctorado). Universidad de Salamanca, Salamanca, España.

Ulu, H., \& Ulusoy, M. (2019). The development of metacognitive awareness of reading strategies through WebQuest based teaching. Pegem Eğitim ve Öğretim Dergisi, 9(3), 765-818. http:// dx.doi.org/10.14527/pegegog.2019.025 